\title{
ARMES ET ARMURES DANS L'OEUVRE ÉPIQUE ET HISTORIQUE DE JEAN D'OUTREMEUSE (XIVe SIÈCLE)
}

PAR

CLAUDE GAIER

JEAN d'Outremeuse fut et reste le plus populaire des vieux auteurs qui ont écrit l'histoire de l'ancienne principauté de Liège. Cette notoriété, il la doit sans doute à l'usage de la langue vulgaire - un français teinté de 'wallon- et aussi à l'extraordinaire profusion de détails pittoresques qui émaillent ses narrations, où vingt générations d'érudits, de littérateurs et d'échotiers du passé ont puisé leur inspiration. Pourtant, la critique historique de notre siècle n'a eu aucune peine à démontrer que pareil ascendant n'était pas mérité, puisque Jean d'Outremeuse avait bel et bien recopié d'autres récits ou inventé de toutes pièces la matière qu'il n'avait pas trouvée dans des sources antérieures. De sorte que, à quelques nuances près, l'admiration sans bornes qu'on lui vouait jadis à désormais cédé la place au scepticisme, voire au dénigrement systématique à son égard ${ }^{1}$.

Cependant, il existe une autre façon d'aborder les écrits de cet auteur: une analyse en quelque sorte au second degré de ses assertions, qui permet de pénétrer son univers d'homme du XIV siècle et, par là-même,

${ }^{1}$ L'oeuvre de Jean d'Outremeuse a donné lieu à une littérature historique et philologique très abondante, dont nous ne citons ici que les principaux titres: A. Borgnet et S. Bormans, Chronique et geste de Joban des Preis, dit d'Outremeuse, t. VII (Introduction), Bruxelles, 1887; A. SCHELER, La Geste de Liège par Jeban des Preis, dit d'Outremeuse. Glossaire philologique, Bruxelles, 1882; S. BALau, Comment Jean d'Outremeuse écrit l'Histoire..., in «Bull. Comm. R. Hist.», t. 71, 1902, pp. 63-95; ID., Les sources de l'bistoire du pays de Liège au Moyen Âge. Étude critique, Bruxelles, 1903, pp. 559-576; G. KuRTH, Étude critique sur Jean d'Outremeuse, Bruxelles, 1910; L. Mrchel, Les légendes épiques carolingiennes dans l'oeuvre de Jean d'Outremeuse, Bruxelles-Liège, 1935; H. Nissen, L'ordre des mots dans la chronique de Jean d'Outremeuse, Uppsala, 1943; J. LeJeune, Jean d'Outremeuse, le quatrième livre du «Myreur des bistors» et la «Cbronique en bref», in «Annuaire d'Histoire Liégeoise», t. IV/4 (1951), 1952, pp. 457-525; A. Goosse, La «Chronique abrégée» de Jean d'Outremeuse, in «Rev. belge Philol. et Hist.», t. 32/1, 1954, pp. 5-50 (voir la polémique entre ces deux derniers auteurs, ibid., t. 34/4, 1956, pp. 985-1020 et 1248-1251); A. Goosse, Jean d'Outremeuse, Ly Myreur des Histors. Fragments du second livre (années 794-826), Bruxelles, 1965, Introduction critique, pp. XLII sv. 
de nous informer sur les réalités de cette époque. Cette approche, nous l'avons réalisée à travers les descriptions de combats et de batailles, extrêmement nombreuses dans l'oeuvre en question, à cause du parti-pris épique et romanesque qui l'anime. L'idée d'exploiter cet aspect particulier de la fiction littéraire n'est pas nouvelle. Des travaux semblables, basés sur les chansons de geste et les romans courtois allemands ${ }^{2}$, français ${ }^{3}$, anglais ${ }^{4}$ et espagnols ${ }^{5}$ ont donné d'excellents résultats, en nous éclairant à la fois sur l'armement ancien et sur la façon dont les auteurs du Moyen Âge ou leurs compilateurs le percevaient.

\section{Les oeuvres de JeAn d'Ou'tremeuse}

Jean d'Outremeuse naquit et vécut toute sa vie (1338-1400) à Liège, ville des bords de la Meuse, actuellement en Belgique et qui était alors

2 A. Schulz, Das böfiscbe Leben zur Zeit der Minnesinger, 2 vol., Leipzig, 1889. Extrait intitulé: Blankwaffen und Rüstung der Minnezeil 11. bis 13. Jabrbundert, republié à Nuremberg, en 1967 (Cbronica. Dokumentation aus allen Zeiten in Worl und Bild für Forscher und Sammler, Folge 51-52-53).

${ }^{3}$ Voir A. Sterniberg, Die Angriffswaften im Altfranzösischen Epos, in «Ausgaben und Abhandlungen aus dem Gebiete der romanischen Philologie», t. 48, 1886, pp. 1-52; V. Schirling, Die Verleidigungswaffen im Alt/ranzösischen Epos, ibíd., t. 69, 1887, pp. 1-86; V. BACH, Die Angriffswaffen in der Alifranzösiscben Artus- und Abenleuer-Romanen, ibid., t. 70, 1887, pp. 1-58; W. GIESE, Waffen nach den provenzalischen Epen und Chroniken des XII. und XIII. Jabrbunderls, in «Zeitschrift für romanische Philologie», t. 52, 1932, pp. 351-405; D. J. A. Ross, L'originalité de «Turoldus»: le maniement de la lance, in "Cahiers de Civilisation Médiévale», t. 6, 1963, pp. 127-138; J. WaT'HELET-WILLEM, L'épée dans les plus anciennes chansons de gesles. Elude de vocabulaire, in Mélanges R. Crozet, t. I, Poitiers, 1966, pp. 435-449.

+ M. L. Keller, The Anglo-Saxon weapon names, Heidelherg, 1906 (Anglistische Forschungen, núm. 15); F. DeTERs, Die Englischen Angriffswaffen zur Zeit der Einführung der Fenerwaffen (1300-1350), Heidelberg, 1913 (Anglistische Forschungen, núm. 38); E. M. Parkinson, Sir Pbilip Sidney on jousting and lilting, in Proceedings of the Ninth Triennial Congress of the International Association of Museums of Arms and Militury History, Washington-New York, 1981, pp. 132-146.

5. W. GIESE, Waffen nach der Spaniscben Literatur des 12. und 13. Jabrbunderls, in Mitteilungen und Abbandlungen aus dem Gebiete der romaniscben Pbilologie, t. 6, 1925; ID., Waffengescbicbuliche und-terminologiscbe Aufscblüsse aus Katalaniscben literariscben Denkmälern des 14. und 15. Jabrbunderts, in Homenatge a Antoni Rubio i Lluch, t. I, Barcelone, 1936, pp. 33-67; M. DE RtQuer, El armamento en el «Roman de Troie» y en la "Hisloria Troyana», in «Boletín de la Real Academia Española», t. 49, fasc. 188, 1969, pp. 643-494; ID., La lecba del «Ronsasvals» y del «Rollan a Saragossa» según el armamenlo, ibíd., t. 49, fasc. 187 , 1969, pp. 211-251; ID., El baubert francés y la loriga castellana, in Mélanges de Pbilolugie et de Lilléralures romanes offerls à Jeanne Walbelel-Willem, Liège, 1978, pp. 545-568; ID., Las armas en el «Amadís de Gaula», in «Boletín de la Real Academia Española», t, 60, fasc. 221, 1980, pp. 331-427. 
la capitale d'une principauté ecclésiastiqué relevant du Saint-Empire germanique. Greffier de l'official, c'est-à-dire du tribunal ecclésiastique, il avait statut de clerc et consacra une bonne partie de son temps à la lecture de chansons de geste, de romans, d'ouvrages didactiques et de chroniques.

Son oeuvre historico-littéraire fut considérable, encore qu'inachevée. De plus, il n'y en a qu'une partie qui soit parvenue jusqu'à nous. Seuls nous intéressent ici «La Geste de Liège» et «Ly Myreur des Histors» (Le Miroir des Histoires), dont cet article constitue l'analyse particulière ${ }^{6}$.

La première est une épopée, à la gloire de la cité de Liège, dont subsistent environ 52.000 alexandrins groupés en laisses monorimes. Elle commence à la guerre de Troie - car la ville de Tongres, d'où naquit l'évêché de Liège, fut prétendument fondée par un fils d'Enéeet s'achève à la fin du XIV ${ }^{c}$ siècle. Le second est une oeuvre de compilation, en trois parties, allant du Déluge à 1340 , bien qu'un quatrième livre, couvrant les événements postérieurs à cette date ait, semble-t-il, existé mais soit aujourd'hui perdu. Le «Myreur» comporte de nombreux fragments dérimés, empruntés par Jean d'Outremeuse aux épopées qu'il avait lui-même composées antérieurement: la «Geste de Liège» dont question ci-dessus, et une «Geste d'Ogier le Danois», à présent disparue. On a d'ailleurs souligné combien la dette de notre auteur était grande envers la matière épique carolingienne. Il fait notamment d'Ogier le Danois, petit-fils de Doon de Mayance, son héros chevaleresque par excellence, personnage de prédilection dont il multiplie à l'envi les attaches - inventées pour les besoins de la cause - avec le pays de Liège ${ }^{7}$ (fig. 1).

\section{LES FAITS D'ARMES DANS L'OeUvRES de JEAN D'OUt'REMEUSE}

Jean d'Outremeuse est avant tout un romancier, qui se sert de l'Histoire - tout au moins de ce qu'il en connait - comme d'une toile de fond, pour y broder des histoires. Sa conception du passé relève, par ailleurs, d'une philosophie manichéenne et simpliste, volontiers moralisatrice: les Hébreux contre les gentils, les chrétiens contre les païens,

"Chronique el geste de Joban des Preis, dit d'Outremeuse, éd. A. Borgne' et S. Bormans, 7 vol., Bruxelles, 1864-1887. Nous hous référerons désormais à la «Geste de Liège» par le sigle $G$ et au «Myreur des Histors» par le sigle M. Pour les fragments du «Myreur» qui se rappottent aux années 794 à 826 , nous renverrons plutôt à l'édition de Gosse, cit. n. 1 ci-dessus, par le sigle M-Gosse.

7 Cf. Michel, Les légendes épiques carolingiennes..., op. cit. 


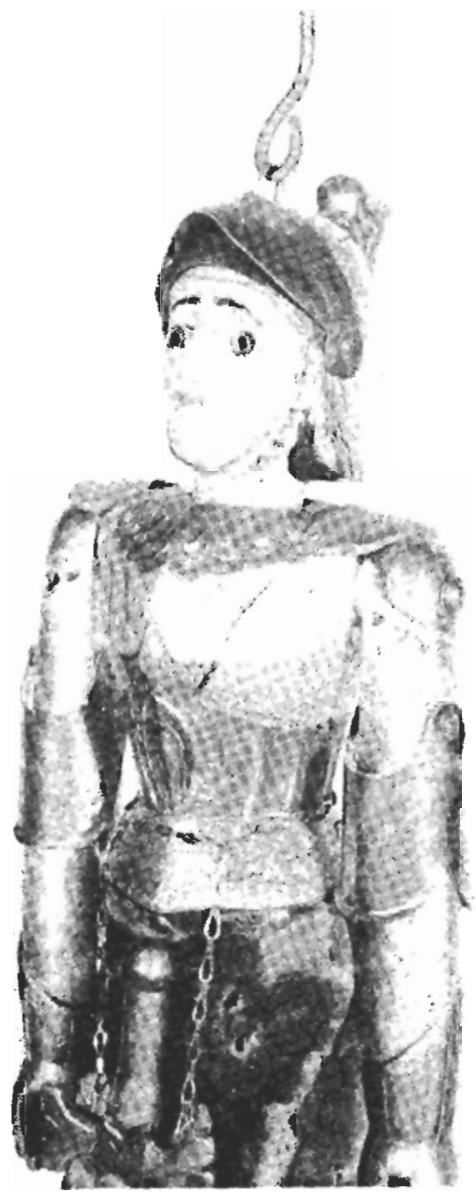

FIG. 1. Ogier le Danois, jeune. Personnage traditionnel du théâtre de marionnettes de Liège; celui-ci puise une partie de son inspiration dans les romans de chevalerie. Collecrions et cliché du Musée de la Vie Wallonne à Liège.

les catholiques contre les hérétiques, les ecclésiastiques contre les laïcs, les preux contre les félons et ... les gens de Liège contre beaucoup d'autres. Bref, les «bons» opposés aux «mauvais». Cette grande soif de justice expéditive et de départage entre le bien et le mal, il l'assouvit 
en multipliant les épisodes violents, les faits d'armes en particulier, où les plus méritants, s'ils ne sont lâchement trahis, reçoivent, par la force qui guide leur bras, la juste rétribution de leur valeur. Les affrontements militaires sont donc très nombreux dans les écrits de Jean d'Outremeuse et c'est surtout dans «La Geste de Liège» qu'ils atteignent les plus longs développements, favorisés par les nécessités verbales de la rime.

$\mathrm{La}$ disparition d'une partie de l'oeuvre relative au XIV $\mathrm{XI}^{\mathrm{c}}$ siècle nous empêche d'apprécier suffisamment la façon dont l'auteur aurait présenté les événements proches de son temps. On se rend compte, cependant, qu'il fait montre d'une plus grande sobriété et d'un certain réalisme dans le traitement des épisodes relativement récents. Est-ce prudence de sa part à l'égard d'éventuels contradicteurs voire de témoins directs? Ou bien considérait-il le romanesque et l'affabulation comme un artifice de «distanciation», applicable seulement à un lointain passé?

A cette nuance près, les affrontements qu'il décrit et leurs acteurs qu'il met en scène reflètent, quelque soit l'époque à laquelle ils se rapportent, deux conceptions distinctes, bien que superposécs, des pratiques guerrières. La première, qui l'emporte de loin, véhicule les clichés littéraires empruntés par l'auteur aux chansons de geste et aux romans courtois: elle présente la guerre comme une juxtaposition de combats singuliers et de prouesses héroïques. Ses archétypes nous ramènent aux $\mathrm{XI}^{\circ}, \mathrm{XII} I^{*}$ et $\mathrm{XIII}{ }^{*}$ siècles, encore que ce parangon chevaleresque à survécu à travers tout le bas Moyen Âge. La seconde conception répercure la vision de Jean d'Outremeuse lui-même, homme du XIV siècle, à une époque où le rapport des forces militaires est en train de changer en faveur de l'infanterie et des formations tactiques plus élaborées. Il ne s'agit point dans ce cas d'un modèle, suivi de propos délibéré, mais plutôt de l'influence, sans doute inconsciente, des réalités contemporaines sur l'auteur. On verra l'incidence de cette dualité sur la connaissance des armes.

Il n'y a donc pas lieu de s'étonner si les guerriers de l'Antiquité ou du haut Moyen Âge ressemblent, sous la plume de notre auteur, à ceux du «Roland» ou de «Perceval» (fig. 2a). Le frère d'Antiochus de Syrie (II" siècle avant J.C.) est présenté comme un «mult bon chevalier», tandis que Jules César «astoit tant poisans, fors et hardis et victorieux, que quant ilh astoit armeis sour unc cheval, et ilb entroit en batalbe, ilh faisoit ses annemis reculeir demy-bonier, et les faisoit fuyr». Sous le bas Empire romain, on lit —entre autres fables - qu'un duc de Gaule nommé Franco «fiert entour de luy de sa bache com bons chevaliers». Décrivant un combat en champ clos -imaginaire- entre deux personnages qui ne l'étaient pas moins: Franbal, roi des Latins, et Alexandre, prince d'Athènes, Jean d'Outremeuse croit utile de préciser qu'il s'agis- 
sait là du «promirs champs qui fut fait en Europe corps à corps». Savoureaux anachronisme, Franbal se pare d'armoiries: de sable à un léo-

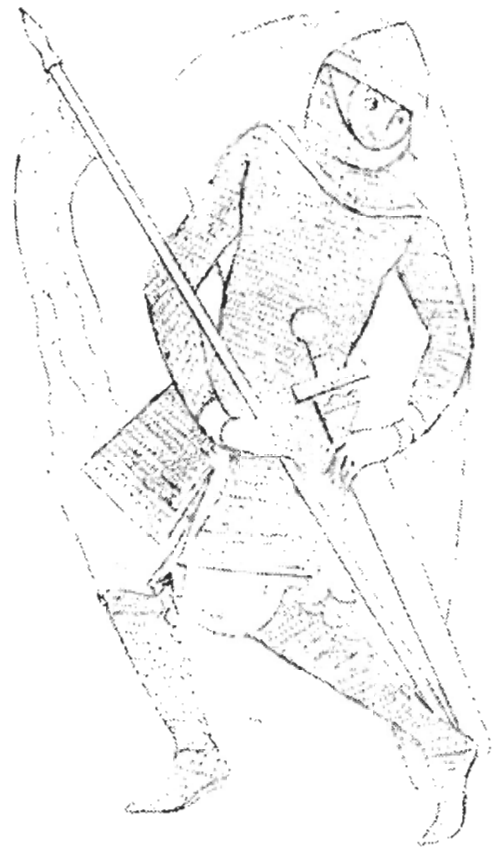

FIG. 2a. L'archétype du guerrier selon Jean d'Outremeuse, celui des chansons de geste et des romans courtois du XII ${ }^{\circ}$ siècle. «Goliath», d'après un Psautier de la Bibliothèque Nationale, à Paris (Lat. 8846). Dessin de Viollet-le-Duc.

pard d'or ${ }^{8}$. Même projection dans le lointain passé à propos d'Ogier le Danois, sorte de surhomme du temps des Croisades égaré à l'époque de Charlemagne ${ }^{9}$.

8 M, I, pp. 130, 144, 155, 219, 547.

- Voir, en particulier, l'éducation chevaleresque et l'adoubement d'Ogier: MGoosse, pp. 20, 32, 39-40. 
Cà et là pourtant - nous l'avons dit plus haut- percent quelques allusions à des pratiques plus proches de celles du XIV siècle. Ogier, au siège de Sébaste, utilise des archers génois et quand il range son armée en bataille pour affronter les Nubiens, il «mist les archiers devant et fist une batailhe a trois pointez». Quant à l'évêque de Liège Otbert, il escorte l'empereur en Italie avec cinq cents chevaliers, chacun accompagné de trois «lances», allusion anachronique aux petites unités tactiques du bas Moyen Âge, composées de plusieurs hommes d'armes. Les trente jouvenceaux qui exécutent le traître Vauquetin se servent d'ailleurs d'«arcs anglais». L'auteur utilise aussi une expression à la mode lorsqu'il parle d'une «rote» (du latin rupla) de Sarrasins, pour désigner un groupe de combattants placés sous le même commandement. Enfin, toujours à propos d'Ogier, il le décrit choisissant cent chevaliers entre cinq cent Hesbignons "qui sont preux et bardis» précise-t-il, afin de combattre le roi païen Agolant, en Espagne ${ }^{10}$. On sait que les hommes d'armes de l'évêque de Liège, qui se recrutaient principalement dans la région naturelle appelée «Hesbaye», aux $X I I I^{e}$ et $X I V^{e}$ siècles, jouissaient alors d'une solide réputation guerrière ". Notons encore une remarque fort pertinente de Jean d'Outremeuse, qui dénote un certain sens du recul historique: à propos d'une campagne de l'évêque de Liège Baldéric, en 1041, contre le duc de Champagne, il signale que le prélat avait emmené dans sa troupe «touz gens d'estat, à cheval; et la pielalhe n'astoit point acostumire d'aleir en ouste». Par ailleurs, lorsqu'il évoque des batailles, en des temps plus proches du sien, notre auteur assigne aux fantassins le rôle important qui est désormais le leur, en particulier dans la principauté de Liège. En dépit de ses préjugés aristocratiques, il se réfère alors fréquemment aux «gens menues» dans ses narrations militaires et il leur prête un armement disparate, composé souvent de haches et de massues, bien conforme à la réalité ${ }^{12}$.

\section{LA TECHNIQUE DU COMBAT SINGULIER}

Comme dans les oeuvres épiques et romanesques du Moyen Âge, Jean d'Outremeuse ramène les récits de batailles à la description d'une successsion de combats singuliers, où les adversaires sont généralement

désignés par leur nom. Ces guerriers, selon le modèle tactique médiéval

${ }^{10}$ M-Goosse, pp. 36, 62, 142, 146; M, III, p. 111; G, II, p. 743, v. 19.112; G, IV, p. 611, vv. 30.123 à 30.125 .

"Cf. C. GaIER, Art et organisation militaires dans l'ancienne principauté de Liège "t dans le comté de Looz au Moyen Âge, Bruxelles, 1968, pp. 182-183.

12 M, IV, p. 224; cf. C. GAIER, op. cit., p. 149. 
et en vertu d'une conception aristocratique de la politique et de la guerre, sont presque toujours des cavaliers, de rang social élevé. Lorsqu'il se lasse de commenter les échanges de coups entre deux héros, ou que son imagination lui fait défaut pour identifier de nouveaux personnages, notre auteur se contente d'alléguer la destruction d'un grand nombre d'ennemis, afin de justifier la victoire de celui ou de ceux qu'il désire faire triompher. Il va de soi qu'il partage la propension des chroniqueurs contemporains pour les chiffres fantaisistes et exagérés, dans le but d'impressionner le lecteur par le niveau des effectifs et des pertes des antagonistes qu'il met en scène.

Les affrontements individuels se déroulent généralement selon les phases stéréotypées qui prévalaient non seulement dans la littérature épique et romanesque mais dans la réalité militaire d'une époque qui avait le culte de la cavalerie lourde.

Pour Jean d'Outremeuse, les adversaires, montés et «armés de pied en cap» ${ }^{13}$, se précipitent d'abord l'un vers l'autre, lance baissée ${ }^{14}$. Il se peut qu'un des hommes soit désarçonné et jeté à bas de sa monture. Ainsi, provoqué en duel par Roland, Ogier le Danois aurait, d'un coup de lance, levé le preux chevalier de six pieds par dessus l'arçon de sa selle ${ }^{15}$. Si l'adversaire renversé est encore en état de lutter, le combat se poursuit, à pied. Il se peut aussi que la victime tienne bon, demeure à cheval, mais soit transpercée, comme le frère d'Antiochus Epiphane, «oultre le corps piet et demy» ${ }^{16}$. Au moment du choc, les lances volent en éclats, en plusieurs tronçons, dont certains restent fichés dans le bouclier ou l'armure des antagonistes. Lors d'une rude mêlée, Ogier le Danois, "XX trons de lanche enporte en son escut, et $X$ en son babier»" L'écu, qui soutient le premier choc, est transpercé, fendu, et le haubert déchiré ${ }^{18}$.

Se protégeant alors du bouclier, ou de ce qu'il en reste, solidement fixé à l'avant-bras gauche ${ }^{19}$, les adversaires tirent l'épée: «Apres les lanches ont sachiés les brans tot nus» ${ }^{20}$. Ils échangent des coups d'estoc

" M, VI, p. 391.

it Voir notamment le combat entre Franbal et Alexandre: M, I, p. 155.

is M-Goosse, p. 108.

16 M, I, p. 130.

${ }_{17} \mathrm{M}$, III, p. 225

${ }^{18}$ G, I, p. 600, v. 963; III, pp. 518-519, vv. 29.445-29.446, 29.484; IV, p. 620, v. $30.743 ; \mathrm{V}$, p. 641 , vv. 4.102 à $4.104 ; \mathrm{M}, \mathrm{I}$, pp. 246-247; IV, p. 316; V, p. 364 Nombreux autres exemples.

${ }_{19}$ M, VI, p. 86: le roi de France, désarçonné, «salbit sus el trait son espée, et le tenoit en son pongne el mist l'escut devant»; M, VI, p. 427: l'évêque de Liège «son escut acollat et tient l'espiel d'achier».

${ }^{20} \mathrm{G}, \mathrm{I}, \mathrm{p} .618$, v. $2.148 ; \mathrm{M}, \mathrm{I}$, p. 532. 
et de taille, plus de cent dit-on parfois ${ }^{21}$. C'est ce que Jean d'Outremeuse appelle «jouer d'escremmerie» ou d'«esquermerie» ${ }^{22}$. À propos d'Ogier et de Palamède, il commente «qui les veist enbrachier leur escus et maniier leur espée del esquerime, geteir de stoch et de tailhe, bien diroit: Chu soni prinches eslues» ${ }^{23}$. Cette forme primitive d'escrime -et qui en porte déjà le nom- privilégiait les coups de taille. Car il est rarement fait mention d'assauts de la pointe, alors que les effets spectaculaires du tranchant, considérablement amplifiés par le ton épique, s'étalent tout au long des récits de batailles: heaumes et coiffes entaillés, ennemis fendus jusqu'aux dents, jusqu'à la poitrine, jusqu'à la selle du cheval, ou séparés en deux, comme une bûche ${ }^{24}$. Les coups les plus terribles sont portés en tenant l'épée des deux mains. Atteinte par un aussi terrible moulinet, la tête d'Alexandre, prince d'Athènes, est projetée à quarante pieds, tandis que les Francs de Charlemagne, qui guerroient en Espagne contre Agolant, se servent ainsi de leur lame pour couper cavaliers et montures d'un seul coup! ${ }^{25}$ Il n'est pas interdit de penser, toutes proportions gardées, que ces descriptions sanglantes de Jean d'Outremeuse s'inspirent non seulement des clichés littéraires habituels mais également de la réalité de son temps. Il faut rappeler ici qu'Olivier de la Marche disait les épées des archers bourguignons capables de sectionner bras et jambes, ou de couper un homme en deux, par le milieu du corps! ${ }^{26}$

Enfin, les combattants à cheval utilisaient généralement une dague, ou «miséricorde», pour achever leur adversaire renversé. Jean d'Outremeuse en parle à plusieurs reprises ${ }^{27}$.

En dehors de la lance, de l'épée ou du couteau, les autres armes apparaissent moins fréquemment dans les descriptions de combats singuliers. Il s'agit alors d'armes de choc. C'est ainsi que certains personnages de marque se servent de la hache: le duc de Gaule, les évêques de Liège Notger ou Adolphe de la Marck, des chevaliers, le sarrasin Robastre ${ }^{28}$... La masse d'armes ou le marteau d'armes apparaissent également dans

" M, I, p. 155; M-Goosse, p. 158

${ }_{22}$ M, I, p. 246; M-Goosse, pp. 158, 195, 219.

"M, III, p. 331 .

2 Par exemple: M, I, pp. 246-247, 547, 548; G, I, pp. 600, vv. 964-965, p. 618, v. $2.164 ; \mathrm{G}, \mathrm{II}$, pp. 564 , v. 5.597 ; p. 663 , vv. $12.942-12.945 ; \mathrm{M}$, III, pp. 119-120, 204; M-Goosse, pp. 17, 100, 108, 113, 143, 151, 165, 169.

¿ M, I, p. 155; M-Goosse, pp. 119-120, 204.

p. 87

${ }^{27} \mathrm{CH}$. Brusten, L'armée bourguignonne de 1465 à 1468, Bruxelles, [1953],

${ }_{27}$ Notamment M, V, pp. 119.120.

${ }^{28}$ M, I, p. 547; IV, p. 172; VI, p. 430; G, III, p. 452, v. 24.203; VI, p. 669, v. 10.616: M-Goosse, p. 11 . 
les mains de guerriers de haut rang: Lyon Sanson, patrice de Rome, des évêques de Liège, ou des nobles pour la première ${ }^{29}$; pour le second, Charles Martel, le roi sarrasin Lucabel ou le héros légendaire Colin Maillart, encore que ce dernier, maçon de son état, utilisait un maillet de fer de dix pieds de long, version fantastique d'un modeste outil de sa profession, aux origines fort peu aristocratiques ${ }^{30}$.

Pour Jean d'Outremeuse, le combat par excellence est avant tout affaire de lance et d'épée, et il n'était assurement pas le seul à penser de la sorte alors que prévalait encore l'image chevaleresque du guerrier.

Il convient maintenant de passer en revue les diverses armes mentionnées par notre auteur et d'étudier le sens qu'il donne aux vocables par lesquels il les désigne. Une première remarque s'impose: les substantifs «arme», «armure» et le verbe «armer» possèdent, sous sa plume, des acceptions qui diffèrent parfois assez sensiblement de celles d'aujourd'hui.

\section{ARMES ET ARMURES}

Contrairement à l'usage actuel, «arme» et «armer» ont le plus souvent un sens défensif: être armé veut dire porter une armure. D'où les expressions: "armeis de tous harnas» ${ }^{31}$ ou "de toutez armeiz» ${ }^{32}$ ou bien "de piet en cappe» ${ }^{33}$. Le contexte, dans bien des cas, éclaire abondamment cette signification:

«il luy at detrenchiet toutez sez armes jusques en chair»

"... fendu li at la targe,

Et le babier fauseit et l'auqueton savage;

Arme dont soit armeis ne ly font avantages

«La gens l'empereur armeis de jaserans»

«... cbescun sens armes ocist VI babregiés»

"... tout oltre le perchoit,

Les plattes et les armes et le cuer li crevoits

${ }^{24}$ M, II, p. 427; IV, p. 225; G, III, p. 490, v. 27.213; p. 509, vv. 28.708 et 28.709; IV, p. 642 , v. 32.383 .

${ }^{3}$ M. II, p. 427; III, p. 234; IV, pp. 169, 191.

"G, I, p. 601 , v. 1.033 ; IV, p. 614 , v. 30.325 .

12 M-Goossc, p. 106.

"M, VI, p. 391. 
"Et ilh ne puet vestir ne broingne ne armes fines, Car oncques ne fut armeis.... ${ }^{34}$.

En corollaire, les «gens d'armes» sont, pour Jean d'Outremeuse, les combattants par excellence, c'est-à-dire les cavaliers cuirassés: "Ilb n'oit nulle pitons en cel oust, ains furent toutes gens d'armes» remarque-t-il à propos de l'armée des coalisés qui chevauche contre le duc de Brabant en $13322^{3.5}$.

Exceptionnellement, «arme» et «armer» se rapportent à du matériel offensif:

«... si prendent cognie et baches, et marteals et teils armes, si deffonsent ches bons tonials à vins...»

"La commone est armeis à hache et à marteals» ${ }^{30}$.

Le cas échéant, le substantif et le verbe peuvent concerner l'héraldique:

"Et estoint Liégoix tous armeis des armes sainct George, une blanche colle avec une rouge croix...» ${ }^{7}$.

Le mot «armure» peut désigner, comme aujourd'hui, un équipement défensif:

«... C chevaliers, très-nobles en armeure»

«... mult bien sunt assenois sour les targes, si ont tout desquire it les armeures, et si passenl oultre sens estre navreis» ${ }^{38}$.

Cependant, il arrive également qu'il revête un sens mixte et qu'il signifie -contrairement à l'usage restrictif actuel — toute espèce d'armes, défensives et offensives:

D'aubry de Montdidier, momentanément privé de son haubert, il est dit qu'«il n'avoit nullez armeurez fors son espée».

"4 Cf. successivement: M-Goosse, p. 165; G, I, pp. 600-601, vv. 963-965; G, IV, p. 715 , v. $37.541 ; G, V$, p. 657 , vv. 5.499 ; p. 659 , vv. $5.606-5.607$; p. 663 , v. 5.933 5.934.

${ }^{15}$ M, VI, p. 492. Aussi l'expression «hommes d'armes» dans M-Goosse, p. 27.

${ }_{16}$ M, V, p. 555; G, VI, p. 641, v. 8.684 .

"Chronique abrégée de Jean d'Oulremeuse, in «Chroniques liégeoises», éd. Balau et Falron, t. II, Bruxelles, 1931, pp. 177.

${ }^{88} \mathrm{G}, \mathrm{V}$, p. 668, v. $6.351 ; \mathrm{M}, \mathrm{IV}$, p. 370. 
Décrivant le trésor d'Ogier, à Meaux, composé de «mult d'armures et de joweaz», I'auteur énumère: «... cent baymes, cent babiers, cent espées.... ${ }^{39}$.

Enfin «armure» avec le déterminatif «de fer», désigne chez Jean d'Outremeuse, selon la coutume générale du XIV ${ }^{*}$ siècle d'ailleurs, un homme d'armes:

«... si furenl bien $X X^{m}$ armeurs de fier de gens esluil...»; une escorte de "cent armurs de fiers»; un chanoine belliqueux, qualifié de "mult valbant armures de fier" ${ }^{40}$.

Le fer désigne d'ailleurs la matière par excellence dont se compose l'équipement défensif, le «harnoi» ${ }^{41}$ :

"... l'evesque asloit jà à Waremme, à $I I I^{\prime \prime}$ armeir de fier bien stoffeis»:

«...IIIc barons armeis et fervestus»;

"A XL" bommes qui los sont fervestus»;

«...li damoiseals, de corocbe esmeüs,

Vinrent jusqu'à la balle des drappiers fervestut» ${ }^{42}$.

\section{LES ARMES DÉFENSIVES}

\section{Le boqueton}

C'est une cotte rembourrée que le chevalier revêt en premier lieu, sous son armure, afin d'amortir les coups. Pièce nécessaire, mais insuffisante par ellc-même pour assurer la protection du combattant, Jean d'Outremeuse la considère comme la base de l'équipement défensif. Il nous montre son héros, Ogier, dépossédé de ses armes par des traîtres

"." M-Goosse, p. 125; M, V, pp. 130, 135.

M, IV, p. 494; VI, pp. 417, 502.

" G, II, p. 647, v. 11.845; "III" of o luy d'bommeis armeis de bon harnas»; G, IV, p. 614, vv. 30.325-30.326: «Ly conte de Savoie, armeis de cous barnas, // Astoil enmy l'estour». Cependant, «harnoi» a parfois le sens d'équipement ou de bagage en général, comme dans le cas suivant (G, V, p. 616, vv. 2.301-2.302): "L'evesque se partit et sens gens aylant, // Leur harnas sont trosseit et vont apparilhant».

${ }^{42} \mathrm{M}, \mathrm{IV}$, p. 578; G, V, p. 619, v. 2.561; G, I, p. 617, v. 2.133; G, VI, p. 658, vv. $9.897-9.898$. 
et réduit à ne disposer que d'un hoqueton pour les affronter. De même, lorsque le petit-fils de Doon de Mayence tombe aux mains des sarrasins, ceux-ci lui enlèvent tout moyen de résister et ne lui laissent que son hoqueton.

Pour abattre un homme d'armes, il faut non seulement percer ou trancher son bouclier et son haubert, mais aussi son hoqueton:

«... Jendu li at la targe,

Et le babier lauseit et l'auqueton savage»;

"La coelle et aqueton, torniquet et parure,

A trestout detrenchiet...»;

"Le duc at assenneit de si noble Jacbon.

Que escut et babert, chemiese et oqueton

Li at tout deromput..." ${ }^{43}$.

\section{Le baubert}

C'est l'armure par excellence des combattants. On discute encore de nos jours de la nature exacte des matériaux qui la composaient et les allusions, extrêmement nombreuses, que Jean d'Outremeuse fait à ce vêtement défensif ne permettent hélas pas de mettre un terme à ce débat. Il s'agit d'une tunique, comportant des manches longues et un capuchon (la coiffe) d'un seul tenant, accompagnée de chausses, qui se mettent séparément ${ }^{\text {H }}$ (fig. 2a). On la désigne ici indifférenment semble-t-il, par les termes «haubert» ou «broigne». Elle est en tout cas faite de mailles -quelque soit le sens que l'on puisse attribuer à cet ancien vocable - que la violence des coups est susceptible d'arracher: «il luy desmailbat sa brongne et le navrat en costeit...». Ou encore «La coeffe desmailbat, si le fiert à plains bras..." ${ }^{4 \bar{j}}$.

Le haubert, la broigne sont parfois qualifiés de dobletin, dobletine ou de trelhie $^{46}$, expressions qui figurent déjà dans les anciennes chansons de geste et qui désignent des variantes dans la construction de ces vêtements défensifs, variantes dont le sens n'est pas clair.

t. M-Goosse, p. 52; M, III, p. 248; G, I, p. 600, vv. 963-964; II, p. 693, v. 15.235; V, p. 641 , vv. 4.102-4.104.

${ }^{44}$ Lors de l'adoubement d'Ogier par Charlemagne, celui-ci présente les diverses pièces d'armement au jeune chevalier; le haubert et les chausses sont distinctement désignées: M-Goosse, p. 40.

4: M-Goosse, p. 100; G, III, p. 519, v. 29.511.

*6 $G, I I I$, p. 518, v. 29.446 ; IV, p. 614 , v. $30.352 ;$ p. 662, v. 33.729 . 


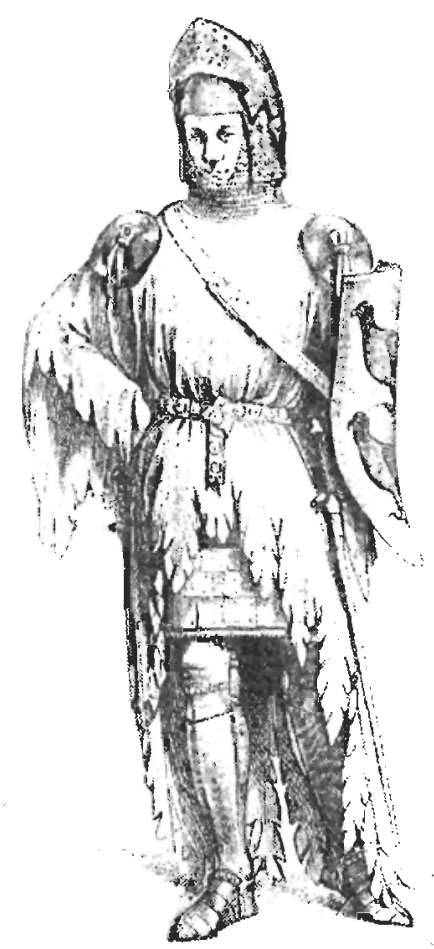

FIG. 2b. Le guerrier tel qu'il était réellement à la fin de la vie de Jean d'Outremeuse. Dessin de Viollet-le-Duc d'après une statuc de Judas Machabée, jadis à Pierrefonds.

Le «jaseran» est une sorte de haubert dont on pense qu'il se composait de plaquettes de métal reliées entre elles par des anneaux de fil de fer. Il est cité quelquefois par Jean d'Outremeuse:

"Le bealme li fausat, qui astoit reluisans, Et la coeffe ansiment del habier jaserans»

"La gens l'emperour armcis de jaserans»

"As cops de lanches ont rompu main jaserant" ${ }^{47}$.

" G, IV, p. 620, vv. 30.742-30.743; p. 715, v. 37.541; G, I, p. 622, v. 2.494. Voir aussi M, III, pp. 267, 284. 
Il faut également signaler que l'auteur attribue parfois à des animaux de guerre une protection en «jaseran»: ainsi le destrier de l'avoué de Saint-Lambert de Liège, ou le cheval du champion sarrasin chargé de se mesurer à Ogier, ou encore les boeufs qui trainnent, sur le champ de bataille, le «beffroi» de bois du roi d'Aragon ${ }^{48}$. Pour le reste, les chevaux sont habituellement présentés domme dépourvus d'armure, si ce n'est la housse de tissu qui parfois les recouvre ${ }^{49}$.

Quant aux hommes, ils revêtaient une cotte d'armes, appelée «tourniquet» ou «talevas» par dessus l'armure ${ }^{50}$.

\section{Le haubergeon}

Cette armure de mailles, qui est sans doute un diminutif du haubert, n'est pas souvent citéc dans notre source ${ }^{31}$. On y utilise également ce terme pour désigner l'homme d'armes ainsi équipé:

$$
\text { "Se sunt partis de Treit IIIC babregons" } " \text {. }
$$

\section{La cuirie}

La cuirie est une défense du tronc faite de cuir bouilli. Bien que celle-ci soit d'un emploi courant au XIV e siècle, Jean d'Outremeuse n'en parle qu'à l'occasion d'épisodes très anciens et exotiques: l'armure du géant Goliath, les guerriers d'Alexandre le Grand montés sur des dromadaires et des chameaux, ou bien les chiens de combat de l'illustre conquérant ${ }^{53}$.

\section{Les plates}

On désignait du nom de «plates», au XIII" siècle, mais surtout au $\mathrm{XIV}^{\mathrm{e}}$, des plaques de fer rivées sur un support de toile ou de cuir, ser-

${ }^{48}$ G, II, p. 762 , v. 20.634 , et M, III, p. 378; M, III, pp. 263, 326.

${ }^{49}$ La housse du cheval de l'avoué de Saint-Lambert est une "covreteur» blanche; M, V, p. 20. Celle du cheval de l'évêque de Liège en 1328 est un "singlaton» vermeil; M, VI, p. 425.

s0 G, I, p. 602, v. 1.042; II, p. 693, v. 15.235; M, V, p. 27. À propos des Liégeois au siège d'Argenteau (1347), la "Chronique en Bref» déclare que les milices communales étaient vêtues d'«une blanche cotte avec une rouge croix», analogue sans doute à celle des chevaliers; Chronique abrégée de Jean d'Outremeuse..., op. cit., p. 177.

"s1 G, IV, p. 662, v. 33.730

$\therefore$ G, V, p. 619 , v. 2.557 .

"G, IV, p. 662, v. 33.729; M, I, p. 281. 
vant à protéger le tronc ou les membres des combattants. Par extension, on appela «plates» l'armure ainsi composée. Jean d'Outremeuse pare quelquefois ses personnages de ce genre de défense à partir du XII ${ }^{\mathrm{c}}$ siècle, anachronisme évident mais qui semblerait indiquer tout de même une certaine conscience du caractère récent de cet armement. Les plates

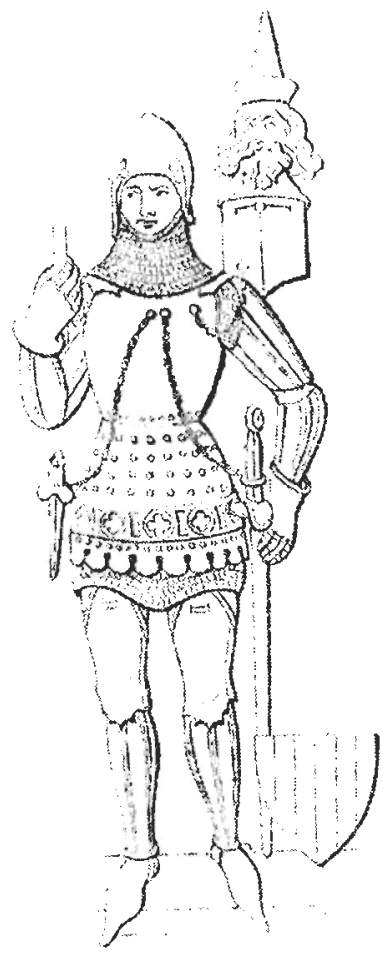

Fic. 3. Effigie funéraire de Conrad de Seinsheim (1369) à Schweinfurt, d'après Hewitt, Ancient $A r$ mour. Les chaînes attachées à la cotte de plates sont bien visibles.

sont ici associées au haubert, ce qui est exact puisque les premières étaient conçues pour renforcer la cotte de mailles ${ }^{54}$.

L'auteur n'ignore pas non plus que l'épée et la dague étaient parfois fixées au plastron de la cotte de plates par des chaînes destinées à retenir ces armes lorsqu'elles glissaient des mains de leur utilisateur (fig. 3).

\$4, III, p. 518 , v. $29.446 ;$ V, p. 659 , v. $5.606 ;$ p. 477 , v. $6.743 ;$ p. 689 , v. $8.232 ; \mathrm{M}, \mathrm{V}$, p. 569 . 
Il rapporte à ce sujet un accident dont fut victime un chevalier français, assiégé dans Cassel par les Flamands, au début du XIV siècle. Alors qu'il précipitait sur les assaillants une grosse pierre, celle-ci se prit «à la chaine de ses plates»s et l'entraîna par son poids en bas de la muraille ${ }^{55}$.

\section{Le beaume}

Le heaume est le casque par excellence, celui qui est le plus fréquemment cité. Il est difficile de savoir comment l'auteur se le représentait, car on retrouve à nouveau dans ses descriptions une superposition entre les modèles de la littérature épique ou courtoise et ceux d'une réalité plus contemporaine.

Des expressions comme "elme à or brunit» ${ }^{56}$, "byalme bruny" ${ }^{57}$, «byalme gemeis» ${ }^{53}$, «byalme à crestale» ${ }^{59}$, «beame agus»" ${ }^{* 0}$, «cercle et nasal" ${ }^{61}$ renvoient à la première catégorie, c'est-à-dire au casque ouvert qui fut seul utilisé jusqu'à la fin du XII" siècle. Par contre, les allusions à l'«elme fermeit" ${ }^{62}$, au "beame à claviel» ou "clavure» ${ }^{63}$ (i. e. à charnière), à la «ventalbe» et à la visière ${ }^{64}$, indiquent que Jean d'Outremeuse songe aussi non seulement au heaume complet qui, depuis le commencement du XIII ${ }^{c}$ siècle, emboîtait toute la tête, mais même à celui, plus perfectionné encore, qui était muni d'une partic mobile afin de découvrir le visage (fig. 4).

Le heaume est surmonté d'un cimier: le timbre; celui d'Ogier le Danois représente un épervier posé sur une couronne d'or ${ }^{65}$.

On trouve également ici le mot «heaume» désignant l'unité de combattant, soit l'homme d'armes coiffé de ce casque, d'usage général dans la terminologie militaire au XIV $\mathrm{Xich}^{\mathrm{c}}$ siècle ${ }^{6 \sigma}$.

\section{Le bassinet}

D'après ce que nous en savons aujourd'hui, le bassinet était un casque hémisphérique ou conique, qui couvrait le crane et la nuque et se

$5 \mathrm{M}, \mathrm{VI}$, p. 15.

:6. G, I, p. 600, v. 929.

" G, I, p. 618, v. 2.204; M-Goosse, p. 195.

${ }^{58} \mathrm{G}, \mathrm{I}, \mathrm{p} .619$, v. 2.276 .

${ }^{59} \mathrm{G}, \mathrm{II}$, p. 546 , v. 4.289 ; III, p. 452 , v. 24.221 .

${ }^{*} \mathrm{G}, \mathrm{II}$, p. 747, v. $19.506 ;$ V, p. 619 , v. 2.583.

A M-Goosse, p. 113

$n$ G, IV, p. 612, v. 30.240 .

G, II, p. 634, v. 10.773; IV, p. 643, v. 32.411; VI, p. 668, v. 10.590.

it G, II, p. 663, v. 12.942; M, VI, p. 391

${ }^{65}$ M-Goosse, p. 40.

M-Goosse, p. 3. 
prolongeait par une pélerine de mailles le camail, protégeant les joues, le menton, le cou et les épaules. Il comportait souvent aussi une visière articulée qui masquait le visage. Mais on donnait aussi le nom de bas-

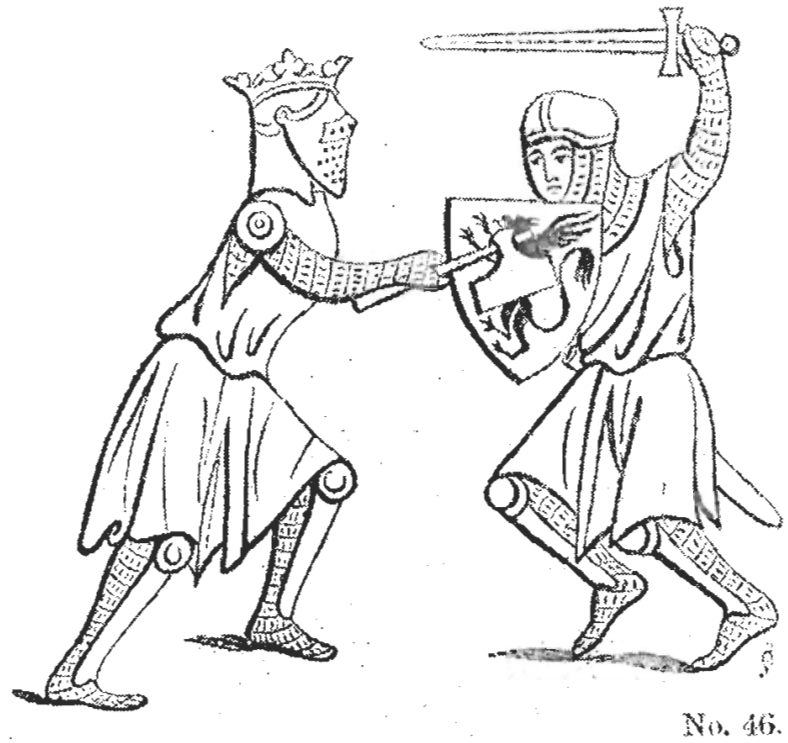

FIG. 4. Combat de chevaliers (début XIV siècle). Celui de gauche porte un heaume à visière, sans doute du type auquel se réfère J. d'Outremeuse. D'après Hewitt, Ancient Armour... (Miniature du British Museum, Roy Ms. 16, G, VI, $\mathrm{f}^{\circ}$ 172).

sinet à un petit casque, sorte de cevellière, qui ne coiffait que le sommet de la tête, et pouvait se porter seul ou sous le grand heaume.

Cette fois, le modèle n'est pas livresque et Jean d'Outremeuse parle non de ce qu'il a lu mais de ce qu'il a pu voir de son temps. Pour lui, le bassinet ( «bachin» ${ }^{67}$, "bachinet») ${ }^{68}$ est bien muni d'un camail ( "barbier») ${ }^{\leftrightarrow}$ et d'une visière ${ }^{70}$; ou encore c'est un petit casque (qu'il appelle

${ }^{67} \mathrm{G}, \mathrm{II}$, p. 564, v. $5.597 \mathrm{sv}$.

G, VI, p. 658, v. 9.866 .

${ }^{n 9}$ G. VI, p. 659 , v. 9.958 .

${ }^{70}$ Le seigneur de Harduémont est tué d'un coup de lance au visage parce que, dit Jean d'Outremeuse, il ne portait ni heaume ni bassinet, ce qui indique claire- 
«bacin» ou «capel» ou «buvette») ${ }^{71}$ qui se porte seul ou sous le heaume. Il le qualifie parfois: «bassin d'assier» ${ }^{72}$. Comme dans le cas du heaume, le terme bassinet peut désigner un combattant, selon l'usage habituel du temps ${ }^{73}$.

\section{Le chapeau}

Le chapeau, coiffure militaire à larges bords, peu usitée dans la cavalerie, est rarement cité. Il est parfois qualifié: «capel d'acier» ${ }^{74}$.

\section{Le bouclier}

La protection que les combattants fixent à l'avant-bras gauche afin de parer les coups est appelée indifféremment écu ou targe par Jean d'Outremeuse. Celui-ci n'établit aucune distinction entre ces deux termes ${ }^{75}$, auxquels les archéologues et historiens modernes attribuent cependant des acceptions quelque peu différentes. Quoiqu'il en soit, le bouclier -écu ou targe - est peint ${ }^{76}$, doré ${ }^{7}$ ou émaillé ${ }^{78}$ et bordé d'un listel $^{79}$. Il se suspend au cou par une courroie ${ }^{80}$. Habituellement en bois, on cite un cas où il est fait de corne et de nerfs collés ${ }^{81}$.

La targe, de surface plus importante, que l'on utilisait notamment pour les travaux de siège, est simplement qualifiée de «grande targe» ${ }^{82}$.

ment que, dans l'esprit de l'écrivain, ce dernier casque était muni d'une visière; M, VI, p. 288 , variante 3 ; cette leçon mentionne «viaire» (visage) au lieu de «ventre», ce qui est de toute évidence plus correct puisque le coup est censé atteindre le cerveau.

${ }_{71}$ Le recours à des synonymes indique que Jean d'Outremeuse attribuait le même sens à ces trois vocables: M-Goosse, pp. 106, 108 («bacin»= «capel»); G, II, p. 571, y. 6.133 («bubette» ou huvette = bassinet).

${ }_{72}$ G, III, p. 508, v. 28.690; IV, p. 662, v. 3.373; M-Goosse, p. 106.

${ }^{73} \mathrm{G}$, IV, p. 639, v. 32.204 (《dois $M$ bacbins»); M, VI, p. 529 («Vc bachines $q u$ 'ilh avoit de ses bans»). Alors que le ms suivi par l'éditeur donne «cent lancbes» à propos d'un contingent armé, une autre leçon cite «cent bachines»; M, VI, p. 258 et variante num. 11.

${ }^{74} \mathrm{G}$, IV, p. 615, v. 30.396; V, p. 590, v. 165; M, III, p. 263.

${ }^{35}$ Cf. notamment G, IV, p. 621, v. 30.854 et 30.857 .

7h $G$, II, p. 724, v. 17.664: «escus poiniureis».

${ }^{n G} \mathrm{G}, \mathrm{III}$, p. 518 , v. 29.445 : «escus à or fins».

${ }^{78} \mathrm{G}, \mathrm{I}, \mathrm{p} .600$, v. 929: «escut à esmale».

${ }^{79} \mathrm{G}, \mathrm{I}, \mathrm{p} .607$, v. 1.400: «escus listeis»; II, p. 683, v. 14.442: «targe listée».

${ }^{*}$ M, IV, p. 495: «le coroie de l'escul».

: G, II, p. 757, vv. 20.256-20.257.

${ }^{82}$ M-Goosse, p. $142 ;$ M, IV, p. 547. 
Enfin, la targe désigne aussi un combattant: au siège de Bouillon (1141), l'évêque de Liège aurait disposé de trente mille «targes» ${ }^{83}$.

Dans un seul cas, l'auteur emploie le mot bouclier, pour désigner sans doute la petite rondelle de poing d'usage moins aristocratique que l'écu: "contre ses cops ne dure byalme, escus ne bocleire" ${ }^{84}$.

Enfin, on trouve aussi le mot «écu» dans un sens héraldique ${ }^{85}$.

\section{LES ARMES OFFENSIVES}

\section{Le bâton}

L'usage de bâtons est moins anodin qu'il y paraît, car ce terme peut en fait désigner toute espèce d'arme offensive, selon l'acception répandue au Moyen Âge et que l'on retrouve chez Jean d'Outremeuse. Ainsi, au cours d'une insurrection en la ville de Huy, le maïeur s'efforce de calmer la foule en lui recommandant de s'abstenir de «cops d'espeez, de bache, espaffus ne d'altre baston». Une anecdote relative à la bataille de Mons-en-Pévèle (1304) est révélatrice sur ce point: le roi de France, un moment désarçonné, ne peut se servir de son épée, qui vient de se briser. Il avise alors un boucher, porteur d'une grande hache, et il lui dit «moy donne ton baston». Il paraît qu'il en fit bon usage pour se dégager de l'étreinte ennemie ${ }^{*}$.

Cependant, à côté du sens d'arme ofensive en général, on rencontre des cas où le mot bâton a la même signification que de nos jours. Ainsi Roland, qui vient de perdre sa fidèle Durandal, se présente au combat armé d'un simple bâton de cinq pieds de long "gros devant». Lorsque le comte de Moha entreprend l'éducation chevaleresque de ses deux fils, il leur déclare: "desormains vous commande le mestier de bastons et de pierches vos ensaieis, mains ne vos blecbiez» et "Si ne prendeis bastons dont vous puissiez blechier». Manifestement, il fait allusion à des exercices physiques inoffensifs, avec des simulacres d'armes. Ces sages conseils n'allaient pas être suivis: un jour, les deux jouvenceaux s'essayèrent à la joute avec des lances de guerre et moururent transpercés... ${ }^{87}$

G, IV, p. 645, v. 32.585.

* G, I, p. 619, v. 2.256.

"Cf. J. J. Hoebanx, Le sens d'«écu» dans un passage de Jean d'Outremeuse, in «Rev belge Philol. Hist.», t. 37, 1959, pp. 695-702.

M, V, p. 554; VI, p. 86.

${ }^{87}$ M-Goosse, p. 154; M. IV, p. 567 (aussi G, V, p. 584, v, 50). Le traître Jean du Pont s'arme d'«I grans bastons de chayne»; G, VI, p. 659, v, 10,003. 


\section{L'épée}

Arme individuelle par excellence, l'épée est naturellement celle qui apparaît le plus fréquemment dans l'oeuvre de Jean d'Outremeuse. Elle comporte une poignée (bue) ${ }^{88}$ avec une garde (enordure) ${ }^{89}$ et une lame (alemele ou bran): "son espee brisat desus le buse et l'alemele volat desus le preit»" ${ }^{\infty}$ " $A$ dois mains tient l'espee dont li brans fut letreis ${ }^{91}$. Elle se range dans une gayne ou foreal ${ }^{92}$.

Employant la partie pour le tout, à l'instar des auteurs d'épopées et de romans, Jean d'Outremeuse désigne souvent l'épée par le mot «bran», avec une parfaite synonymie ${ }^{93}$. Toujours inspiré par la même veine littéraire, il donne à cette arme des appellations d'origine stéréotypées: "d'ovraige de Saxangne»" "brans poitevins» "5, "espee sardine»", «brans vienois» ${ }^{97} .$. Quant à l'expression «espée de Bale» ${ }^{98}$, il pourrait s'agir, par contre, d'une allusion à un article d'exportation connu au XIV' siècle.

Les qualificatifs attribués aux épées ou aux parties de l'épée trahissent également des réminiscences de lectures: "qui d'or a l'enordure 99 (garde)», «brans... letreis» ${ }^{100}$, "brant d'achier brunit» ${ }^{101}$, "boin brant de colour» ${ }^{102}$, "brant d'achire» ${ }^{103}$, "boin brant de metale» ${ }^{104}$, "espee forbie» ${ }^{105}$, «qui ot longe allemel» ${ }^{106}$, «qui talbe oltre mesure» ${ }^{107} \ldots$ preit».

${ }^{*}$ M, VI, p. 86: «son espee brisal desus le buse et l'alemele volat desus le

${ }^{84} \mathrm{G}, \mathrm{I}$, p. 634, v. 10.772: «Ilh tint l'espée nue qui d'or a l'enordure...».

*) Ibid.

"G, VI, p. 659, v. 9.976 .

"2. M, VI, p. 119.

9: Par exemple, la «Geste» et le «Myreur» utilisent indifféremment «bran» ou «épée» à propos du même épisode; $G, V$, p. 631, v. 3.542, et $M, V$, p. 228. Cf. aussi M-Goosse, p. 216.

${ }^{\circ} \mathrm{G}, 1$, p. 602 , v. 1.071 .

:5. G, III, p. 518, v. 29.456 .

* G, IV, p. 628, v. 31.354 .

${ }^{97}$ G, II, p. 747, v. 19.503 ; G, IV, p. 640, v. 32.272 .

*? G, I, p. 600, v. 949.

${ }^{9}$ G, II, p. 634, v. 10.772: «Ilh tint l'espée nue qui d'or a l'enordure...".

${ }^{100} \mathrm{M}, \mathrm{V}, \mathrm{p}$. 86: «son espee brisat desus le buse et l'alemele volat desus le preil».

${ }_{101} G$, II, p. 752, v. 19.855 .

$102 \mathrm{G}, \mathrm{I}$, p. 618 , v. 2.164 .

${ }^{103}$ G, I, p. 618, v. 2.204

${ }^{104} G, I I$, p. 560 , v. 5.315 .

10. G, IV, p. 662 , v. 33.731

1n6 $\mathrm{G}, \mathrm{V}, \mathrm{p} .689$, v. 8.186

$107 \mathrm{G}, \mathrm{V}$, p. 638, v. 3.883 . 
Enfin, conformément à la grande tradition épique, il existe des épées fameuses, qui portent un nom propre: celle de Roland s'appelle «Durandal» et celle d'Ogier «Courtaine».

\section{Le "faucbon»}

Le "faucbon» (ou malchus) était une petite épée à lame asymétrique et à un seul tranchant qui servait essentiellement à donner des coups de taille. Souvent illustrée à partir du XIII siècle, elle apparaît rarement sous la plume de Jean d'Outremeuse. Cet auteur ne l'attribue qu'à des assassins: ceux de saint Lambert, le patron de Liège, martyrisé vers 705 et dont un tableau très connu du $\mathrm{XV}^{\mathbf{c}}$ siècle représente un des persécuteurs ainsi armé (fig. 5), ou bien les meurtriers d'Abert de Louvain $(1192)^{108}$.

\section{Le couteau}

Le couteau (cutel) ${ }^{109}$ est fréquemment cité et Jean d'Outremeuse le place dans les mains de toutes les catégories de combattants ou d'agresseurs. Il n'établit pas de distinction apparente entre l'outil domestique et l'arme de guerre. C'est ainsi que le mot «dague» n'apparaît pas. Par contre, il utilise parfois le terme «misericorde» pour désigner, seion l'usage de son temps, un poignard destiné à porter le coup de grâce. Ainsi Ogier, qui vient de renverser Roland, veut le frapper à mort en se servant d'une telle arme; Charlemagne, lui aussi, porte une «miséricorde» ${ }^{110}$. Par contre, Ogier se saisit d'un «cutel» pour achever le roi sarrasin Agramart et le comte de Saint-Pol agit de même envers Ferrand de Flandre qu'il vient de renverser ${ }^{111}$.

Il semble donc bien que la terminologie de Jean d'Outremeuse soit ici un peu floue, ou qu'il préfère simplement utiliser le vocable le plus général.

\section{La bacbe}

Arme de choc fréquemment citée, l'auteur ne la distingue pas clairement de la cognée, à usage domestique. En effet, si, à propos de la ré-

${ }^{103} \mathrm{M}, \mathrm{II}$, p. 369 (voir fig. 5 ci-jointe), et G, IV, p. 720, v. 37.933.

${ }^{107}$ Par exemple, M, III, p. 95.

${ }^{110} \mathrm{G}, \mathrm{IV}$, p. 620 , v. 30.771 (aussi ibid., v. 32.703); M-Goosse, p. 27.

"M, III, p. 95; V, p. 120. 


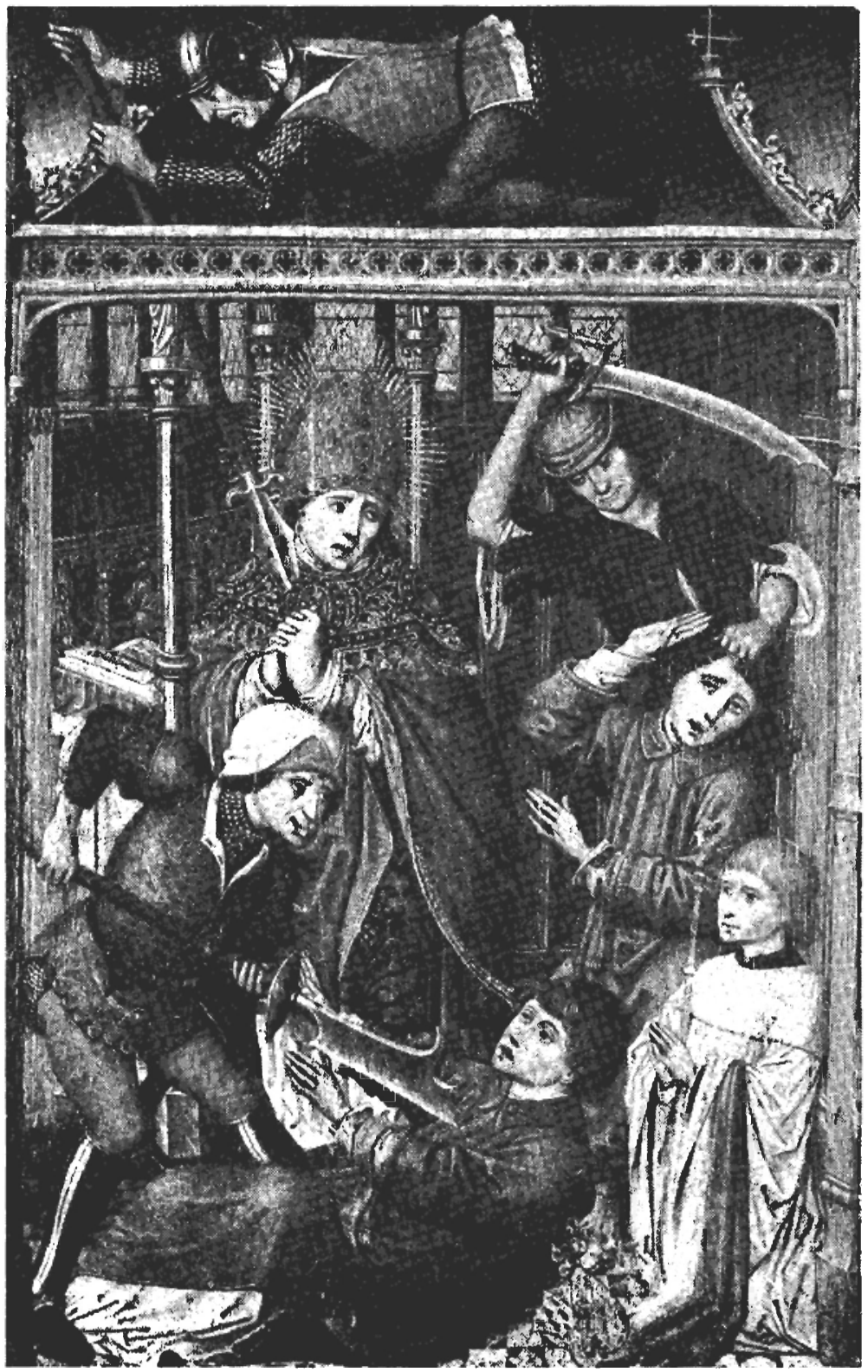

Fıg. 5. Représentation liégeoise du martyre de saint Lambert (fin $\mathrm{XV}^{*}$ siècle). Comme dans la tradition, rapportée par J. d'Outremeuse, un des meurtriers brandit un badelaire. Liège, Musée d'Ar't Religieux el d'Art Mosan. Cliché 3 I. Mascart. 
volte du peuple de Huy, en 1298, il déclare: "si prendent cognie el bacbes, el marleals el teils armes, si diffonsent ches bons tonials a vins...»-passage où l'on peut croire qu'il établit une différence entre les deux - ce n'est pas le cas de l'épisode où il met en scène Robastre, tuant des Sarrasins «de sa cognie ou baicbe» ${ }^{112}$. Pourtant, il n'ignore pas le sens du mot "cognée» puisque, dans un passage qui s'inspire d'une chronique latine, il utilise ce terme afin de traduire sa source qui emploie "dolabra»"

Ces armes ont souvent de grandes dimensions: c'est pourquoi on les manie à deux mains. Celle que le roi de France brandit ainsi à la bataille de Mons-en-Pévèle (1304) "II pies avoil d'allemelle», et celle d'un «Sarrasin» au siège de Paris de 522 était "granl el pesanle» ${ }^{114}$. Ce grand modèle paraît bien être désigné par l'expression «hache danoise», encore qualifiée, à cause de la forme de son fer en croissant de lune, de «mult ague» 115 . Originaire des pays nordiques, cette arme fut utilisée jusqu'au $\mathrm{XIV}^{\mathrm{c}}$ siècle dans toute l'Europe (fig. 6) mais c'est un modèle plus petit, à manche court, qui finit par la supplanter. Toujours avide de passes d'armes retentissantes, il est normal que notre auteur accorda plus d'importance à celle dont les coups trouvaient, sous sa plume, le plus spectaculaire écho.

\section{Le marleau}

Le marteau est une arme répandue, surtout à l'usage des roturiers: "La commone est armeis à bacbe el à marleals». Mais certains hauts personnages s'en servent aussi: Charles Martel —dont le surnom provient de son arme favorite-, le roi sarrasin Lucabel, Colin Maillart, héros légendaire dont le marteau (parfois appelé «maillet») ne mesure pas moins de dix pieds! ${ }^{116}$

Dans un cas, l'auteur fait allusion au umartel que ons nomme piques», probablement le «marteau picois», c'est-à-dire celui dont le mail

$112 \mathrm{M}, \mathrm{V}$, p. 555; M-Goosse, p. 11.

11.3 G, V, v. 6.744; la source latine est la Chronique de Jean de Hocsem (cf. éd. G. Kurth, Bruxelles, 1927, p. 82). On trouve dans un ms du «Myreur», l'expression bache d'armez, que Jean d'Outremeuse n'a pas coutume d'utiliser, mais il s'agit peut-être de l'interprétation d'un scribe pour «bache danois», que l'auteur cite quelquefois; cf. M, VI, p. 438 et variante 9.

114 M, VI, p. 86; M-Goosse, p. 230.

${ }^{115} \mathrm{G}$, VI, p. 676, v. 11.066. Exemples de haches danoises: G, IV, p. 616, v. $30.451 ;$ p. 640 , v. 32.277 ; V, p. 656 , v. $5.354 ; \mathrm{M}$, VI, pp. $86,430,438$.

${ }_{116} \mathrm{G}$, VI, p. 641, v. 8.684 ; M, II, p. 427; III, p. 234; IV, pp. 169, 191; G, III, p. 463 , v. 25.076 
est adossé d'un long ergot aigu. Il le met dans les mains d'un boucher flamand en lutte contre les Français ${ }^{117}$.

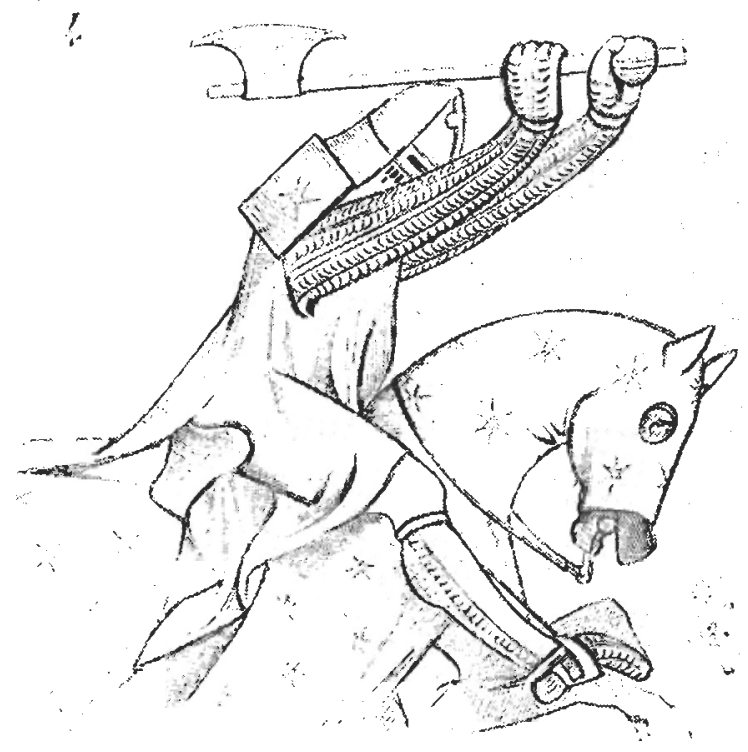

FIG. 6. Chevalier maniant la hache à deux mains. Dessin de Viollet-le-Duc d'après un Roman de la Table Ronde (début XIV' siècle) de la Bibl. Nat. à Paris.

\section{La masse ou massue}

L'auteur semble utiliser les termes «masse» et «massue» indifféremment ${ }^{118}$ et en faire l'arme aussi bien des nobles que des roturiers ${ }^{119}$. Il

${ }^{11 \pi} \mathrm{M}, \mathrm{V}, 507$.

${ }^{118}$ Cf. G, III, p. 509, vv. 28.708 et 28.710.

${ }^{119}$ Usage par l'évêque de Liège: G, III, p. 490, v. 27.213; M, IV, p. 225; par les comtes de Laroche et de Moha: G, III, p. 509; vv. 28.708 et 28.710; G, IV, p. 642 , v. 32.383 ; par des nobles: G, V, p. 682 , v. 7.657 ; VI, p. 669 , v. 10.635 ; M, VI, p. 430; par des roturiers: G, IV, p. 646, v. 32.623; p. 647, v. 32.739; V, p. 607 , v. 1.622 ; p. 608 , v. 1.699 ; p. 656 , v. 5.342 ; p. 657 , v. 5.453 ; VI, p. 653 , v. 9.547 . 
arrive que le métal constitutif soit précisé: «macbe de plonke» ${ }^{120}$ ou "macbue...d'achier» ${ }^{121}$. Un synonyme, «tynal» ${ }^{122}$, est également utilisé pour désigner ce genre d'arme.

\section{Le gourdin}

L'auteur le désigne par les vocables «bourle» ${ }^{123}$ ou «waroquias» ${ }^{124}$. Il en fait une arme de paysans et ne lui attache manifestement guère d'importance. Une version plus noble, le «wangnepain» ${ }^{125}$, est une massue de bois utilisée dans les tournois.

\section{Le "goedendag»}

Arme de prédilection des Flamands, en rébellion contre le roi de France, Jean d'Outremeuse en parle lorsqu'il narre les guerres de Flandre. En connaissait-il la nature exacte? On sait que la définition de cette arme a fait couler beaucoup d'encre ${ }^{12 \cdot}$. Certains auteurs anciens se sont précisément basés sur le passage où notre romancier parle du «goedendag» pour en inférer que celui-ci était muni d'un fer tranchant: un seigneur «portait I gudendaxhe qu'ilh tranchoit tou le fier» ${ }^{127}$. Or, la tendance moderne est de considérer que cette arme devait plutôt ressembler au plançon à picot, c'est-à-dire à un bâton ferré muni d'une pointe. Dans ce cas, le passage incriminé dénoterait plutôt une ignorance de l'objet en question de la part de notre auteur.

\section{La lance}

Nous avons dit plus haut l'importance que Jean d'Outremeuse accordait à la lance en fonction de la place prépondérante qu'elle occupait dans la tactique chevaleresque. Il utilise trois termes pour la désigner:

${ }^{1201}$ G, V, p. 682, v. 7.657.

${ }^{21}$ G, V'I, p. 669 , vv, $10.635-10.636$.

${ }^{122} \mathrm{M}$, II, p. 427 ; VI, p. 86.

${ }^{123}$ M, VI, pp. 258, 262; G, VI, p. 669, v. 10.662

${ }^{12}+M, V I$, p. 57. On trouve également les termes, sans doute synonymes, de: «pan» (G, V, p. 657, v. 5.452), «pesie» (VI, p. 669, v. 10.663, et M, VI, p. 262) et «bredar» $(G, V$, p. 607 , v. 1.623$)$.

${ }^{125} \mathrm{G}, \mathrm{V}$, p. 607 , v. $1.622 ; M$, VI, p. 57.

${ }^{126}$ Cf. bibliographie dans C. GAIER, Les armes, Turnhout, 1979, p. 18, n. 3.

${ }^{127}$ M, VI, p. 85. Cf. J. van MALderghem, La question du Goedendag, in «Ann. Soc, Arch. Bruxelles», t. 10, 1896, pp. 389-390; J. T. DE RAADT, La chevalerie s'estelle servie clu «Goedenclag»?, ibid., p. 394. 
lance («lancbe»), épieu («espiel») et «glaive», et ceux-ci sont, en l'occurrence, strictement synonymes, puisque l'auteur emploie manifestement l'un pour l'autre. Ainsi, entre beaucoup d'exemples, après avoir déclaré que le sire de Herent vient de plonger sa «lance» dans le corps d'Eustache de Herstal, il déclare: "Li espiés est brisiés...». Ailleurs, au sujet du bâtard de Vermandois, armé d'un «glaive», il est dit qu'il «fiert de sa lanche» ${ }^{12:}$. Il semble que ces vocables désignaient aussi la pique, c'est-à-dire la lance de l'infanterie. Jean d'Outremeuse applique en effet les termes: "lance», "épieu» ou "glaive» à l'arme longue des gens de pied ${ }^{124}$, et se sert rarement du mot «pique» ${ }^{130}$. On peut également constater que la lance est parfois utilisée comme projectile, et non tenue sous le bras pour le choc ${ }^{131}$. Cet usage était pourtant tombé en désuétude au $X I V^{*}$ siècle et il ne peut s'agir ici que de réminiscences de la littérature épique. Celle-ci refflétait une situation antérieure où la lance était encore brandie à bout de bras et projetée vers l'adversaire (fig. 7).

Enfin, on notera que «lance» et «glaive» s'emploient parfois dans le sens d'unité tactique de cavalerie:

"Car Vc chevaliers de sanc et de substanche, Des queis cascun avoit aveque luy trois lanche, At l'evesque asembleit...»"

Une curieuse redondance de la «Chronique en Bref», dérivée de Jean d'Outremeuse, nous éclaire tout à fait sur cette acception, largement répandeu à la fin du Moyen Âge: «allat à grande puissance, scavoir 800 lances chevauchie sour l'archevesqué de Coullongne et avoit avec luy VIIIC glaives» ${ }^{133}$.

\section{L'épieu}

En dépit de son habituelle synonymie avec le mot «lance», le terme «épieu» désigne parfois spécifiquement l’arme de chasse caractéristique

${ }^{1 \times}$ G, V, p. 639 (aussi p. 619, vv. 2.576-2.577); M, VI, p. 57. Synonymie également dans M-Goosse, pp. 218, 260.

${ }_{124} \mathrm{G}, 1 \mathrm{~V}$, p. 615 , v. 30.439 sv.: "Les bommez el les jemmez, à espec et espois, // Lancbez, machez et dars et bons archons turquois // Issent de la citeit...». G, VI, p. 675, v. 10.991: "Adont vinrent Ligois a haches et espois». G, V, p. 657, v. 5.453: «A glaives ef à macbucs...». Un notable, défenestré en 1346, tombe sur les glaives des milices communales; Chronique abrégée, op. cit., p. 172.

Ii" Un exemple dans M, IV, p. 311, n. 10: «pickes ef lanches».

131 M, II, pp. 48, 109; III, pp. 221, 225; G, II, p. 758; v. 20.330; IV, p. 673 , v. 34.501 .

${ }_{1.12} \mathrm{G}, \mathrm{IV}, \mathrm{p} .611, \mathrm{vv}, 30.123-30.125$

"in Chronique abrégée, op. cit., p. 229. 
qui porte, de nos jours encore, ce nom. Dans ce cas, le contexte éclaire le sens. Ainsi, Baudouin de Flandre «l'espiel porte ens en pongne, et va cacbier al bois de Noion...» Apercevant un sanglier, il «...prent l'espiel à II mains et le mettit à son piet» afin de ferrer la bête ${ }^{134}$.

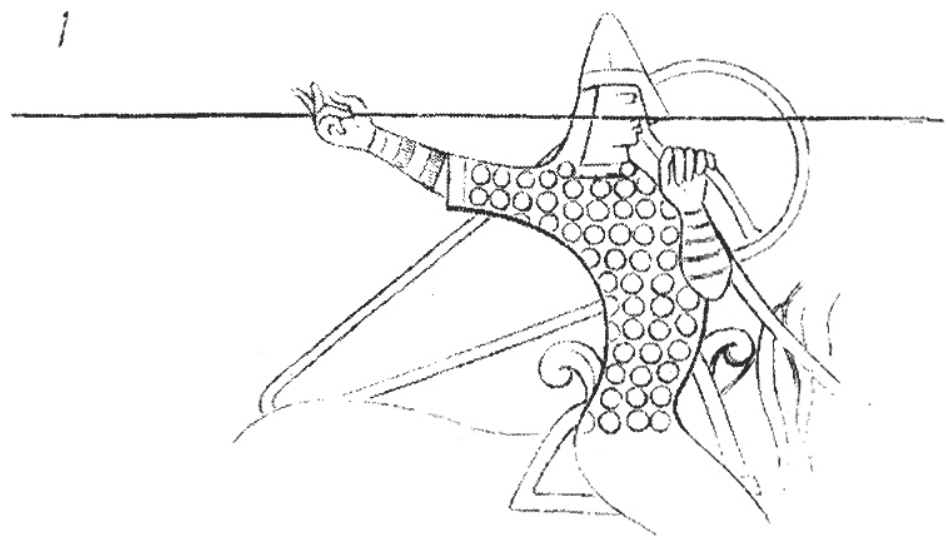

FIG. 7. Maniement ancien de la lance: dardée à bout de bras. Dessin de Viollet-le-Duc d'après la «Broderie de Bayeux», contemporaine de la «Chanson de Roland".

\section{La guisarme}

La guisarme est cette arme de hast bien connue à la fin du Moyen Âge, qui consistait en un fer asymétrique, prolongé par un dard et adossé d'ergots, emmanché sur une hampe. Elle permettait de frapper d'estoc et de taille. Jean d'Outremeuse en parle fréquemment, surtout sous le vocable "spafut» ou «espafut» ${ }^{135}$, dont la signification exacte s'éclaire dans le passage suivant: "onne ghisarme qu'il portoit, que ons nommoit altrement unc spafut» ${ }^{136}$. Les anachronismes sont ici fréquents car l'arme apparaît, avec une invraisemblable précocité, dans les mains du roi des

${ }^{134} \mathrm{M}, \mathrm{IV}, \mathrm{p} .511$.

1.3 Par exemple: M, V, p. 505: «I espafut toute bendeit de fier»; G, VI, p. 668, v. 10.583: «l'espafus qui ot large almial».

${ }_{136} \mathrm{M}, \mathrm{II}, \mathrm{p} .106 . \mathrm{M}, \mathrm{VI}, \mathrm{p} .59$, où une variante dans la tradition manuscrite donne «gisarme» au lieu d' «espafut». Le terme "guisarme» est également utilisé par Jean d'Outremeuse: G, IV, p. 643, v. 32.423; p. 667, v. 34.102; M, IV, p. 311; VI, pp. 20,88 . 
Francs Clodius (IV" siècle) aussi bien que dans celles des guerriers de l'évêque de Liège Notger ( $X^{c}$ siècle) ${ }^{137}$. Sauf exception, la guisarme est employée dans l'infanterie et, le plus souvent, par des roturiers.

\section{Le fauchard}

Autre arme de hast apparentée à la guisarme, le fauchard en diffère cependant par une lame à tranchant convexe. Elle est ici beaucoup plus rarement citée, cependant. On l'attribue également, à vrai dire avec peu de vraisemblance, à des personnages de haut rang: Philippe de Pavie, l'évêque Henri de Gueldre, ainsi qu'aux Romains, ce qui est une pure fantaisie de l'auteur ${ }^{139}$.

\section{Le javelot}

Le javelot ("dard», "lanchelin») "39 est une arme de jet à laquelle l'auteur fait fléquemment rérérence à propos de tous les événements historiques qu'il évoque: les guerres romaines, les Francs (Pépin le Bref reçoit deux javelots dans la cuisse), Charlemagne, les Sarrasins à Damiette, les annales liégeoises ${ }^{143}$. C'est une arme de roturier, bien que l'on cite, au début du XIII" siècle, un chevalier, Bérard de Bruxelles, "qui jettoit dars envinemeis", expression qu'il faut sans doute prendre dans un sens purement figuré ${ }^{141}$.

L'emploi d'armes de jet comme celle-ci donne une fois de plus à Jean d'Outremeuse l'occasion d'exprimer sa conception aristocratique de la guerre car il n'en décrit généralement l'usage que lorsqu'il s'agit de venir à bout d'un ennemi dont la vaillance interdit l'approche au corps à $\operatorname{corps}{ }^{142}$.

L'arc

Arme très répandue, l'arc n'a certes pas la faveur de Jean d'Outremeuse, plus séduit par les instruments de prouesses moins roturières. Il mentionne les arcs «turquois» ${ }^{143}$ et «anglais» ${ }^{144}$, alliant une fois de

13) M, II, p. 263; G, III, p. 447, v. 23.847.

${ }^{13 *}$ G, IV, p. 614 , v. 30.377 ; V, p. 642 , v. $4.122 ;$ M. II, p. 48 .

1.1. Lanchelin est ulilisé pluss rarement; of. par exemple, G, II, p. 707, v. 16.344.

Id1 M, I, p. 144; II, p. 419; G, II, p. 707, v. 16.344; M, III, pp. 221, 345;

V, p. 282; G, IV, p. 646 , v. 32.623 , p. 703 , v. 36.701.

${ }_{1+1}$ M, V, p. 21 .

12 Par exemple M, III, p. 221 (Ogier le Danois).

1.2 G, IV, p. 615, v. 30.440 .

"M-Goosse, p. 62. 
plus les acquis de son érudition et ceux de l'expérience vécue. Cependant, quand il montre le Hun Cymbal tirant, sur le Franc Mérovée, des "saeles barbues», on est porté à croire qu'il n'use pas d'un cliché, mais qu'il se réfère peut-être à ce qu'il a appris de l'armement du peuple de la Steppe ${ }^{145}$.

\section{L'arbalèle}

L'arbalète est souvent citée à l'occasion des sièges ${ }^{146}$ ou dans la dotation des milices urbaines ${ }^{147}$. Visiblement, cette arme est assez familière à notre chroniqueur. Il lui arrive de mesurer une distance en "arbalestrée», comme on le faisait de son temps ${ }^{148}$ et d'utiliser l'expression «rois que quarel» (raide comme un carreau) ou «traire roidemens», par allusion à la force de pénétration du carreau d'arbalète ${ }^{149}$.

\section{LE MATÉRIEL DE SIÈGE}

A côté des armes individuelles, offensives et défensives, Jean d'Outremeuse fait souvent référence à du matériel de siège. Il cite presque toujours celui qui était en usage de son temps, quelle que soit l'époque à laquelle il l'applique, à l'exception toutefois des armes à feu, qu'il ne mentionne pas avant 1346, époque de leur apparition dans la région de Liège ${ }^{150}$.

Les seules notes de dépaysement qu'il introduise dans ses récits concernent le «miroir ardent» et le feu grégeois. Le premier, qu'Alexandre

45 M, II, p. 112. Les «saietes» ou flèches sont encore citées dans M, VI, p. 282: à Damiette, les Sarrasins "commencharent à traire de saietes et de dars si menutment que ploive».

${ }_{1+6} \mathrm{Au}$ siège de Castelfort, où se trouve Ogier le Danois: M, III, p. 249. Au siège de Bouillon (1141): M, IV, p. 384, et G, IV, p. 673, v. 34.546. Au siège de Wijck (1303): G, VI, p. 646, v. 9.053

${ }_{1+7}$ Les bourgeois de Lanson: G, II, p. 707, v. 16.344. Les milices de Tongres: $\mathrm{G}, \mathrm{V}, \mathrm{p} .603$, v. 1.212. Le lignage des Preis, à Liège: $\mathrm{M}, \mathrm{V}, \mathrm{p} .326$. Les bourgeois de Fosses: G, VI, p. 641, v. 8.689. Les arbalétriers de Liège: M, VI, p. 428.

${ }^{148} \mathrm{Cf} . \mathrm{G}, \mathrm{I}$, p. 620, v. 2.363: "Sont Flamens reculeis plus d'une arbalestrée». G, III, p. 462, vv. 25.025-25.026: l'évêque Notger «se trabit avant à une arbalestrée // Près de ses anemis.....

${ }^{149} \mathrm{G}, \mathrm{V}$, p. 638 , v. 3.811; G, VI, p. 641, vv. 8.689-8.690

150 Première mention dans la Chronique abrégée..., op. cit., p. 172, à propos du siège du château de Hamal, près de Tongres, par les milices liégeoises: «'́cs bombards de Harstal et Rocourt...». 
le Grand utilise pour concentrer sur ses adversaires les rayons du soleil, consiste en une surface réfléchissante composée de mille miroirs de fer blanchi et poli ${ }^{151}$. Quant au second, il est dit qu'à la même époque «ilh est une lontaine ès partyes d'orient de cuy aighe ons fait le leu grigois, qui cst si ardent que ons ne le puet estindre, se cbu n'est par vinaigre ou urine d'bomme et savelbon [sable], laqueile ons vent grant argent». Le feu grégeois est encore cité à d'autres occasions, mais à propos de guerres anciennes ou lointaines ${ }^{152}$.

Les moyens de siège comportent la réalisation des travaux d'approche et l'utilisation de dispositifs d'assaut ou de défense.

Dans la première catégorie, l'auteur mentione les terrassements ${ }^{153}$, la sape à coups de pics et de marteaux et le comblement des fossés avec des fagots ${ }^{154}$, l'escalade au moyen d'échelles ${ }^{155}$. Dans la seconde catégorie, il signale le déversement, du haut des murailles, de pierres, de poix brûlante, de chaux vive et de métal en fusion ${ }^{156}$, l'emploi de tours fixes ou mobiles ("engins», "castel», «belefroit») en bois ${ }^{157}$ ou d'un «chat» muni d'un bélier ${ }^{15}$, l'usage de pontons pour franchir une riviè$\mathrm{re}^{15 \%}$ : la mise en batterie de catapultes à contrepoids («engiens», "martinés», "mangonealx», "trébucbets») ${ }^{160}$ ou à arc ("espringales», «quilaine») ${ }^{161}$. Jean d'Outremeuse s'exprime clairement et familièrement au sujet de tout ce matériel, ce qui donne à penser qu'il le connaissait bien et en comprenait le fonctionnement, même s'il puisait ses renseignements dans des récits plus anciens. Sa relation du siège de Lille par le roi de France, en 1296, dénote un entendement particulier en la matière.

159 M, I, pp. 281-282.

152 M, I, p. 293. Ct. aussi le jet de feu grégeois par une machine placée sur une tour d'assaut devant Castelfort, sur ordre du nécromancien Maurin; $M$, III, p. 227 .

153 M, VI, p. 225 (siège du châtcau de Hermalle, en 1315).

is M, III, p. 223.

135 M-Goosse, p. 142; M, III, p. 223; G, V, p. 603, v. 1.226.

in M-Goosse, p. 142; M, III, p. 223; G, V, p. 595, v. 540, et p. 603, vv. 1.224, 1.266; Chronique abrégée, op. cit., p. 179.

${ }_{157}$ M, III, pp. 227, 246; V, p. 329; G, IV, p. 640, v. 32.230; p. 646, v. 32.674.

${ }_{158} \mathrm{M}, \mathrm{V}, \mathrm{pp} .174-175$ : «... ilh list laire I instrument de bois que ons appelloit I cal, à qucile on traiboil le lengne el le terre por remplir les losseis». Cf, aussi G, VI, p. 666, vv. 10.446-10.447 et 10.490-10.495.

${ }_{199} \mathrm{M}, \mathrm{III}$, p. 246.

1*0 M-Goosse, pp. 167, 225; M, I, p. 504; IV, p. 271; V, pp. 164, 174, 186, 200; G, IV, p. 639 , v. 32.182 ; p. 644 , v. 32.482 ; p. 726 , v. 38.289 ; V, p. 657, ․ 5.388; Chronique abrégée, op. cit., pp. 167, 172, 179, 206

in G, IV, p. 727, wv. 38.389-38.390: «... /ait drechier une belle quilaine // C'on "ppelle espringalle en paiis d'Aquitaine...» M, IV, p. 521; VI, p. 83. Il est aussi question de frondes («/ondianes» ou "fonderne») à propos de la bataille de Monsen-Pévèle (1304); M, VI, p. 83. 
Le souverain demande à son «maistre engingnier» de lancer un projectile vers la salle ou se trouve Robert de Béthune. «Atant at fait sa bughe emplir de plonc, et fist sus traire l'engien, et desclichat son clichet, si montal la berge, et li pire s'envolle, si chaiit en la cheminée de la saule... ${ }^{162}$. On ne saurait mieux décrire, en si peu de mots, la manoeuvre d'un trébuchet. Il faut garder à l'esprit, à ce propos, que la guerre de siège était, au Moyen Åge, la plus fréquente et la plus spectaculaire et que les événements politiques survenus à Liège, à l'époque où vivait notre auteur, lui ont souvent donné l'occasion d'entendre parler et peutêtre de voir ces machines belliqueuses ${ }^{163}$.

\section{CONCLUSION}

Jean d'Outremeuse n'est pas un praticien du métier des armes. Il en décrit l'usage à la manière d'un observateur extérieur, qui se réjouit certes à leur spectacle, mais qui n'a aucune expérience personnellc de leur maniement. Toutefois, son souci d'information n'est point négligeable. L'esprit farci de romans chevaleresques, il emprunte à ses modèles les modes de combats les plus spectaculaires et les plus stéréotypés, donc les types d'armements les plus courants. Il les applique en toutes circonstances, sans se soucier des anachronismes, mais il enrichit, plus ou moins consciemment, cet arsenal d'origine livresque en y ajoutant des éléments directement inspirés des réalités militaires de son temps. Ces apports hétérogènes donnent à ses écrits une apparence de réalisme et une vraisemblance qui fit longtemps illusion sur la véracité de ses narrations. En fait, il greffe, sur une trame conventionnelle d'objets, en apparence immuables et sans cesse évoqués -l'épée, la lance, le bouclierune série d'autres instruments - plus spécifiquement contemporains ceux là- dont il se sert pour étoffer sa matière prétendument historique. Cette seconde source d'inspiration reflète la connaissance qu'un lettré du XIV siècle pouvait avoir de l'armement de son temps. Celle-ci n'est point complète, ni pour autant superficielle. L'analyse dénote une vue correcte des grandes catégories d'armes offensives et défensives, mais avec des imprécisions ct des lacunes dans le vocabulaire, car celui-ci ne reflète pas toutes les nuances que nous révèlent l'archéologie ou des sources plus explicites. Si l'on veut bien nous pardonner l'audace de ce rapprochement, la connaissance de l'armement que l'on retire de la lecture de Jean

${ }_{162} \mathrm{M}, \mathrm{V}, \mathrm{p} .508$.

Int Jean d'Outremeuse sait, par exemple, que les catapultes de la ville de Liège étaient construites sur l'île aux Hochets; Chronique abrégée, op, cit., p. 167. 
d'Outremeuse est analogue à celle acquise aujourd'hui en lisant la presse quotidienne, rédigée par des grens "généralement bien informés» qui ne sont pas forcément des experts.

L'étude de notre vieux chroniqueur-romancier a ceci de salutaire et de tonifiant qu'elle nous arrache aux classifications et aux catégories parfois trop abstraites de la science contemporaine, pour nous replonger dans une perception plus directe, plus naturelle, des réalités vécues et des opinions reçues. 